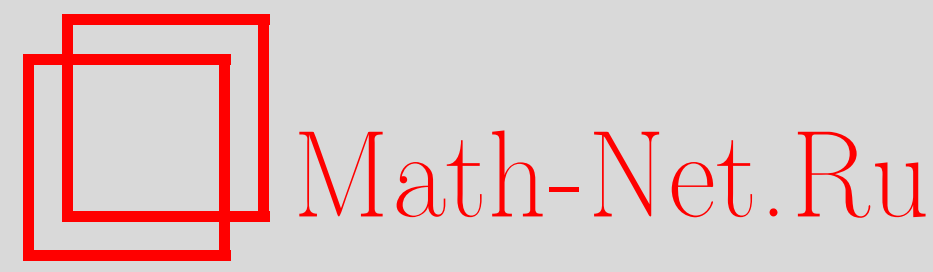

Ю. В. Кузьмин, Группы заузленных компактных поверхностей и центральные расширения, Матем. сб., 1996, том 187, номер 2, 81-102

DOI: https://doi.org/10.4213/sm110

Использование Общероссийского математического портала Math-Net.Ru подразумевает, что вы прочитали и согласны с пользовательским соглашением

http://www . mathnet.ru/rus/agreement

Параметры загрузки:

IP: 18.234 .197 .8

26 апреля 2023 г., $16: 43: 12$ 
УДК 512.04

\author{
Ю.В. Кузьмин
}

\title{
Группы заузленных компактных поверхностей и центральные расширения
}

\begin{abstract}
В статье дается гомологическая характеризация групा, допускающих задание с помощью определяющих соотношений вида $x_{\alpha}^{-1} x_{\beta} x_{\alpha}=x_{\gamma}^{\varepsilon}\left(x_{*}-\right.$ образующие, $\varepsilon= \pm 1$ ). Важность таких групп для геометрии связана с тем, что конечно определенные группы рассматриваемого класса - это в точности групшы заузленных в $\mathbb{R}^{4}$ компактных поверхностей.

Библиографйл: 15 названий.
\end{abstract}

\section{§1. Формулировка основных результатов}

Если некоторый класс груп задан геометрически, естественно попытаться дать его описание в чисто алгебраических терминах. В качестве примера можно вспомнить исчерпывающее решение этой задачи для кристалографических групп или для конечных групп, порожденных отражениями. Хорошо известна проблема алгебраической характеризации групп зацеплений и узлов, которая, однако, до сих пор не решена. Лишш начиная с размерности $n \geqslant 3$ получены необходимые и достаточные условия для того, чтобы абстрактная группа была изоморфна группе $n$-мерного зацепления (соответственно, $n$-мерного узла). Напомним формулировку этого результата, принадлежашего М. Керверу [1].

Пусть $n \geqslant 3$. Группа $G$ изоморфна группе $n$-мерного зачепления тогда и только тогда, когда она конечно определена, совпадает с нормальным замыканием подмножества, которое линейно независимо по модулю коммутанта, и $\mathrm{H}_{2} \mathrm{G}=0$.

В случае $n$-мерных узлов нужно потребовать, чтобы группа $G$ была нормальньм замьканием одного элемента. Тогда условие линейной независимости означает, что факторгруппа по коммутанту $G / G^{\prime}=G_{a b}$ бесконечная циклическая.

Узлы и зацепления строятся с помощью отображений сферы. Как изменится ситуация, если рассматривать и другие компактные многообразия? В. Куликов доказал следующую теорему [2].

Пусть $n \geqslant 2$. Группа $G$ изоморфна фундаментальной группе дополнения $к$ $n$-мерному компактному многообразию, гладко вложсенному в $\mathbb{R}^{n+2}$, тогда

Работа выполнена при финансовой поддержке Российского фонда фундаментальных исследований (грант № 940101575) и Международного научного фонда (грант № MDL 300).

(C) Ю.В. Кузьмин 1996 
и только тогда, когда она имеет конечное копредставление $G=\langle X \mid R\rangle$ с определяющими соотношениями вида

$$
x_{\alpha}^{-1} x_{\beta} x_{\alpha}=x_{\gamma}^{\varepsilon} \quad\left(x_{\alpha}, x_{\beta}, x_{\gamma} \in X ; \varepsilon= \pm 1\right) .
$$

В частности, любая такая группа может быть реализована с помощью вложения компактной поверхности в четырехмерное пространство. Группы, допускаюшие копредставление с соотношениями вида (1), Куликов предложил называть С-группами (по первой букве слова conjugation).

С-группа называется конечно определенной, если соответствующее копредставление конечно. По разным поводам и под другими названиями С-группы встречались во многих работах (см., например, [3]-[7]). Они являются естественньгм обобшением групп одномерных узлов, заданных копредставлением Виртингера [8].

Если ограничиться ориентируемыми многообразиями, то в теореме, приведенной вьше, нужно рассматривать соотношения (1) только с $\varepsilon=1$. Соответствующие С-группы будем называть ориентируемыми. Для ориентируемых С-групп сформулированная теорема была доказана В. Куликовым [9] и, независимо, Дж. Хауви и Н. Гильбертом [10]. Отметим также, что вложениям связных многообразий соответствуют неприводимые С-группы. По определению С-группа $G$ неприводима, если для любых образуюших $x, x^{\prime}$ ее копредставления либо $x$, либо $x^{-1}$ сопряжен с $x^{\prime}$. Из соотношений (1) следует, что это имеет место тогда и только тогда, когда $G_{a b} \cong \mathbb{Z}$ или $G_{a b} \cong \mathbb{Z}_{2}$.

В принципе сформулированная теорема решает вопрос о характеризации фундаментальных групп рассматриваемых пространств. Есть однако некоторое неудобство. Если задана абстрактная группа, то не ясно, как можно узнать, имеет ли она копредставление с определяюшими соотношениями вида (1). Нам удалось получить ответ в терминах, неожиданно близких к теореме Кервера.

В работе [11] была обнаружена естественная связь С-групп с центральными расширениями и второй группой гомологий. Как было замечено в работе [10], если $G$ - неприводимая ориентируемая С-группа, то $H_{2} G$ является фактором централизатора $C(x)$ образуюшего $x \in X$ ее копредставления. Основной результат предлагаемой статьи состоит в том, что последнее свойство можно использовать для характеризации С-групп и, следовательно, для характеризации фундаментальных групп соответствующих дополнений. Чтобы дать точную формулировку, введем несколько определений.

Напомним, что если $G$ - произвольная группа, то любым двум ее перестановочньм элементам $g, h$ соответствует элемент $g \wedge h \in H_{2} G$. В стандартной резольвенте $g \wedge h$ задается циклом $(g \mid h)-(h \mid g)$. Можно дать и другую интерпретацию. Представим $G$ в виде факторгруппы $G=F / N$ свободной группы $F$. Тогда по формуле Хопфа $H_{2} G \cong N \cap F^{\prime} /[N, F]$, а $g \wedge h$ - это коммутатор $[\bar{g}, \bar{h}]$ произвольных прообразов элементов $g$ и $h$ в группе $F /[N, F]$.

Пусть $C(g)$ - централизатор элемента $g \in G$. Тогда отображение $\rho_{g}: C(g) \rightarrow$ $H_{2} G$, определенное равенством $\rho_{g}(h)=g \wedge h(h \in C(g))$, является гомоморфизмом. Как легко следует из определений, образ $\rho_{g}$ зависит лишш от класса сопряженности элемента $g$. Понятно также, что для любого подмножества $B \subseteq G$ определен 
гомоморфизм

$$
\rho_{B}: \bigoplus_{g \in B} C(g) \rightarrow H_{2} G
$$

ограничение которого на прямое слагаемое $C(g)$ совпадает с $\rho_{g}$.

Элемент $g \in G$ назовем ориентируемым, если $g$ и $g^{-1}$ не сопряжены, и неориентируемым - в противном случае. Для подмножества $B \subseteq G$ обозначим $B^{+}$множество его ориентируемых, а $B^{-}-$неориентируемых элементов. Если $h^{-1} g^{-1} h=g$, то $[h, g]=g^{2}$, поэтому неориентируемый элемент либо принадлежит коммутанту, либо его образ в $G_{a b}$ имеет порядок 2. Будем говорить, что множество $B \subseteq G$ линейно независимо с учетом ориентации по модулю коммутанта, если образы в $G_{a b}$ элементов из $B^{+}$линейно независимы в обычном смысле, а образы элементов из $B^{-}$линейно независимы $\bmod 2$. Теперь мы можем сформулировать основной результат.

Tеорема 1. Группа $G$ является С-группой тогда и только тогда, когда она совпадает с нормальным замыканием подмножсества $B \subseteq G$, которое линейно независимо с учетом ориентачии по модулю коммутанта, и отображсение $\rho_{B}: \bigoplus_{g \in B} C(g) \rightarrow H_{2} G-$ эпиморфизм.

Нетрудно доказать, что С-группа $G$ ориентируема тогда и только тогда, когда $G_{a b}-$ свободная абелева группа, т.е. в ориентируемом случае требуется просто линейная независимость.

Понятно, что зная определяющие соотношения группы $G$, легко указать образующие для $\mathrm{H}_{2} G$. Особенность теоремы 1 состоит именно в том, что удается сделать вывод о соотношениях, исходя из информации о группе $H_{2} G$.

Отметим один частный случай теоремы 1.

СлЕДСТВИЕ 1. Пусть группа $G$ совпадает с нормальным замыканием подмножества, которое линейно независимо с учетом ориентации по модулю коммутанта, и пусть $\mathrm{H}_{2} G=0$. Тогда $G$ является $\mathrm{C}-$ группой.

Если группа $G$ конечно определена, а соответствующее подмножество линейно независимо в обычном смысле, то для вывода следствия 1 теорему 1 можно и не использовать. Действительно, тогда по теореме Кервера $G$ - группа 3 -мерного зацепления. Применяя теорему Куликова-Хауви-Гильберта, получим, что $G$-конечно определенная ориентируемая С-группа. Однако этот вывод основан на некоторых глубоких топологических результатах. В теореме Кервера используются, например, результаты Смейла о многомерных гомотопических сферах. Напротив, доказательство теоремы 1 чисто алгебраическое и использует лиш простые факты о центральных расширениях.

Предположим, что множество $B$, о котором говорится в теореме 1 , существует, и пусть $X$ - множество элементов сопряженных с элементами из $B$. Мы докажем, что в качестве определяющих соотношений групшы $G$ относительно системы образуюших $X$ можно взять все соотношения вида

$$
a^{-1} b a=c^{\varepsilon} \quad(a, b, c \in X ; \varepsilon= \pm 1)
$$


которые выполнены в группе $G$. Такое копредставление конечно лишь в том случае, когда конечно множество $X$. Это бывает довольно редко, так как тогда центр группы $G$ имеет конечный индекс. Тем не менее мы докажем, что если группа $G$ конечно определена, то можно указать конечное подмножество $X_{0} \subseteq X$ такое, что соотношений вида $(1)$, где $a, b, c \in X_{0}$, достаточно для задания группы $G$. Другими словами, имеет место

ТЕОРема 2. Конечно определенная группа является С-группой тогда и только тогда, когда она - конечно определенная С-группа.

Для ориентируемого случая теорема 2 была доказана в работе [11]. В следующем параграфе мы укажем, какие дополнения нужно внести в общем случае. Будет также описана процедура фактического построения множества $X_{0}$.

Сопоставляя теоремы 1 и 2 с теоремой Куликова, получим

СлЕДСТвИЕ 2. Пусть $n \geqslant 2$. Группа $G$ изоморфна фундаментальной группе дополнения $\kappa$-мерному компактному многообразию гладко вложенному в $\mathbb{R}^{n+2}$ тогда и только тогда, когда группа $G$ конечно определена, совпадает с нормальным замыканием подмножсества $B \subseteq G$, которое линейно независимо с учетом ориентации по модулю коммутанта, и отображсение $\rho_{g}: \bigoplus_{g \in B} C(g) \rightarrow H_{2} G-$ эпиморфизм.

Другие следствия теоремы 1 обсуждаются в последнем параграфе. Там мы рассматриваем неприводимые ориентируемые С-группы, т.е. те, которые соответствуют вложениям связных ориентируемых многообразий. Дана характеризация таких групп, имеющих абелев коммутант (см. также [11]). Вводятся операции прямого и свободного произведения (они не совпадают с одноименньми операциями в классе всех групп). Описано поведение центральных расширений. Наконец, приведены конкретные примеры, показывающие как работает теорема 1.

\section{§2. Доказательство теорем 1 и 2}

Сушественную роль в доказательстве играет понятие универсального С-накрытия. В ориентируемом случае эта конструкция была введена в работе [11]. Здесь нам понадобится также и неориентируемый вариант.

Пусть $G$ - произвольная группа, $Y$ - ее подмножество и $Y^{G}$ - множество элементов, сопряженных с элементами из $Y$. Будем называть $Y G$-инвариантным, если $Y^{G}=Y$. Пару $(G, Y)$ назовем пред-С-группой, если подмножество $Y$ порождает группу $G$ и $G$-инвариантно. Пред-С-группе $(G, Y)$ можно сопоставить некоторую С-группу $(G, Y)^{*}$ или, для краткости, просто $G^{*}$. Именно, в качестве образующих группы $G^{*}$ возьмем множество $X$ элементов $x_{g}$ индексированных элементами $g \in Y$, а в качестве соотношений - все соотношения вида

$$
x_{a}^{-1} x_{b} x_{a}=x_{c}^{\varepsilon} ; \quad a, b, c \in Y, \quad \varepsilon= \pm 1, \quad a^{-1} b a=c^{\varepsilon} .
$$

Очевидно, отображение $x_{g} \rightarrow g$ продолжается до эпиморфизма $\sigma: G^{*} \rightarrow G$. Группу $G^{*}$ и эпиморфизм $\sigma$ будем называть универсальным С-накрытием пред$\mathrm{C}$-группы $(G, Y)$. Если $H=\langle T \mid R\rangle$ - произвольная С-группа и $\varphi: H \rightarrow G$ - гомоморфизм такой, что $\varphi(T) \subseteq Y$, то, как следует непосредственно из определений, 
отображение $t \rightarrow x_{\varphi(t)}(t \in T)$ продолжается до гомоморфизма $\varphi^{*}: H \rightarrow G^{*}$. Очевидно, диаграмма

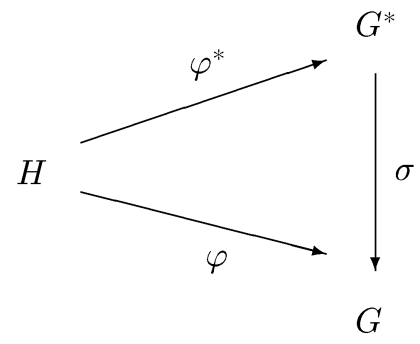

коммутативна. Любая С-группа изоморфна $G^{*}$ для некоторой пред-С-групшы $(G, Y)$. Действительно, пусть $G=\langle X \mid R\rangle$ - некоторая С-группа. Можно предполагать, что $X-$ подмножество групшы $G$. Пусть $Y=X^{G}$, и пусть в диаграмме $H=G$, а $\varphi$ - тождественное отображение. Тогда очевидно $\varphi^{*}=\sigma^{-1}$ и $G \cong G^{*}$.

Пусть $(G, Y)$ - произвольная пред-С-группа. Универсальное С-накрытие $G^{*}=$ $(G, Y)$ можно задать следуюшей системой соотношений

$$
\begin{aligned}
x_{a}^{-1} x_{b} x_{a} & =x_{c} ; \quad \\
x_{c^{-1}} & =x_{c}^{-1} ; \quad c, c^{-1} \in Y, c \in Y, \quad a^{-1} b a=c
\end{aligned}
$$

Действительно, если выполнены соотношения (4), (5), и для некоторых $a, b, c \in$ $Y a^{-1} b a=c^{-1}$, то, поскольку $Y G$-инвариантно, $c^{-1} \in Y$ и $x_{a}^{-1} x_{b} x_{a}=x_{c^{-1}}=$ $x_{c}^{-1}$, т.е. выполнены и соотношения (3). Наоборот, предположим, что имеет место (3), и $c, c^{-1} \in Y$. Тогда из равенства $c^{-1} c^{-1} c=c^{-1}$ вытекает, что $x_{c}^{-1} x_{c^{-1}} x_{c}=$ $x_{c}^{-1}$, и значит, $x_{c}^{-1}=x_{c^{-1}}$.

Рассмотрим следуюшее отношение эквивалентности на множестве $Y$

$$
g \sim g^{\prime} \Longleftrightarrow h^{-1} g h=g^{\prime} \text { или } h^{-1} g^{-1} h=g^{\prime} \quad\left(g, g^{\prime} \in Y, h \in G\right),
$$

и пусть $B$ - множество представителей классов эквивалентности.

Лемма 1. Образ множества $B$ в группе $G_{a b}^{*}$ является ее системой образующих, линейно независимой с учетом ориентации.

ДокАЗАТЕЛЬСтво. По модулю коммутанта соотношения (4) означают, что $x_{a}=x_{c}$, если $a, c \in Y$ и $a, c$ сопряжены, что дает свободную абелеву группу с базой из классов сопряженности, содержащихся в $Y$. Если такой класс $S$ состоит из неориентируемых элементов, то для $c \in S$ соотношение (5) можно переписать в виде $2 S=0$. Если же $S$ состоит из ориентируемых элементов, то множество элементов, которые к ним обратны, образуют класс сопряженности $S^{-1}$, отличный от $S$, а равенство (5) отождествляет $S$ и $S^{-1}$. Это очевидно доказывает лемму 1. 
Ядро универсального С-накрытия $\sigma: G^{*} \rightarrow G$ - это в точности препятствие, для того чтобы группа $G$ была С-группой с множеством образующих $Y$. Мы собираемся дать описание ядра. В качестве первого шага докажем, что ядро лежит в центре. Пусть $v \in G^{*}$ и $w=\sigma(v)$. Тогда для любого образующего $x_{g} \in X(g \in Y)$

$$
v^{-1} x_{g} v=x_{w^{-1} g w} .
$$

Действительно, при $v=x_{h}^{ \pm 1}$ (7) следует непосредственно из определяющих соотношений. Далее можно действовать по индукции: если равенство (7) справедливо для $v=v_{1}$ и $v=v_{2}$, то оно справедливо и для $v=v_{1} v_{2}$.

Из (7) следует, что $v x_{g}=x_{g} v$ в том и только том случае, когда $w g=g w$. Это доказывает следуюшую лемму.

Лемма 2. Прообраз центра группы $G$ при отображении $\sigma$ совпадает $c$ чентром группь $G^{*}$. В частности, $G^{*}$ - центральное расширение группы $G$.

Можно также ввести ориентируемьй вариант универсального С-накрытия, исключая из определяюших соотношений (3) те, в которых $\varepsilon=-1$. Мы будем обозначать универсальное ориентируемое $\mathrm{C}$-накрытие $G_{+}^{*}$. Иногда будем пользоваться и тем же самым обозначением $G^{*}$, если из контекста ясно, какой вариант имеется в виду. Очевидно определен естественньй эпиморфизм $G_{+}^{*} \rightarrow G^{*}$ и для любой пред-С-группы $(G, Y)$ диаграмма

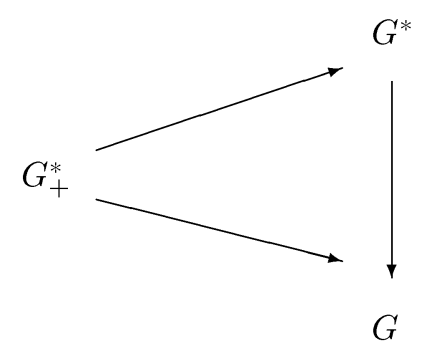

коммутативна. Пусть $D$ - множество представителей классов сопряженности $S \subseteq Y$. Из определяюших соотношений групшы $G_{+}^{*}$ очевидно следует, что ее факторгруппа по коммутанту - свободная абелева с базой, состояшей из образов элементов $g \in D$.

ЛЕмма 3. С-группа $G$ является ориентируемой С-группой тогда и только тогда, когда группа $G_{a b}-$ свободная абелева.

ДокАЗАТЕльство. Представим С-группу $G$ в виде $G=(G, Y)$ для некоторого $G$-инвариантного множества образующих $Y$. Осталось доказать, что, если $G_{a b}-$ свободная абелева группа, то С-группа $G$ ориентируема. Введем сначала соотношения (4). Из леммы 1 следует, что в множестве $Y$ нет неориентируемых элементов, поэтому каждое соотношение (5) отождествляет элементы, индексы которых принадлежат разньм классам сопряженности $S, S^{-1} \in Y$. Мы не изменим группу, если удалим из ее копредставления образующий $x_{c^{-1}}$, где $c^{-1} \in S^{-1}$, удалим 
соотношение (5), а в каждое соотношение (4), в котором встречается $x_{c^{-1}}$, подставим вместо него $x_{c}^{-1}$. Проделав такие преобразования, получим копредставление ориентируемой С-групшы.

Лемма 2 и ее доказательство остаются без изменения для ориентируемого С-накрытия. Установим связь между группами $G_{+}^{*}$ и $G^{*}$.

Лемма 4. Пусть, как и в лемме 1, В -множсество представителей классов әквивалентности по отношению (6), и пусть $B_{0}$ - множсество элементов $с \in B$ таких, что $c^{-1} \in Y$. Тогда ядро естественного отобрахсения $G_{+}^{*} \rightarrow G^{*}-$ свободная абелева группа со свободными образующими $x_{c} x_{c^{-1}}$, где $c \in B_{0}$. Это чентральная подгруппа, имеющая тривиальное пересечение с коммутантом.

ДокАЗАТЕльство. Как мы знаем, группа $G^{*}$ определяется соотношениями (4) и (5). Соотношения (4) задают $G_{+}^{*}$, поэтому ядро отображения $G_{+}^{*} \rightarrow G^{*}$ порождается элементами $x_{c} x_{c^{-1}}$, где $c, c^{-1} \in Y$. Так как ядро центрально, то для любого $g \in Y$ имеем

$$
x_{c} x_{c^{-1}}=x_{g}^{-1}\left(x_{c} x_{c^{-1}}\right) x_{g}=x_{g^{-1}} x_{g^{-1} c^{-1} g} .
$$

Отсюда следует, что, если $c$ и $d$ сопряжены, то $x_{c} x_{c^{-1}}=x_{d} x_{d}{ }^{-1}$, поэтому ядро порождается элементами $x_{c} x_{c^{-1}}$, где $c \in B_{0}$. Эти элементы линейно независимы в факторгруппе по коммутанту, так как ее базу составляют образы представителей классов сопряженности множества $Y$.

Если число классов, сопряженности содержащихся в множестве $Y$, бесконечно, то группы $G^{*}$ и $G_{+}^{*}$ не имеют конечной системы образующих, так как тогда не является конечно порожденной даже факторгруппа по коммутанту $G_{a b}^{*}$. Если же число классов сопряженности в $Y$ конечно и группа $G$ конечно порождена, то $G^{*}$ и $G_{+}^{*}$ - также конечно порожденные группы. Для доказательства достаточно выбрать прообразы конечной системы образующих группы $G$ и добавить к ним множество представителей классов сопряженности, содержащихся в $Y$.

Теорема 3. Пусть $G$ - конечно определенная группа, $Y$ - ее G-инвариантное подмножество, распадающееся в обгединение конечного числа классов сопряжснности. Тогда соответствующее универсальное С-накрытие, как ориентируемое так и неориентируемое, является конечно определенной C-spynnoй.

Для ориентируемого случая эта теорема была доказана в работе [11]. В соответствии с леммой 4 в неориентируемом случае надо добавить конечное множество соотношений $x_{c^{-1}}=x_{c}^{-1}\left(c \in B_{0}\right)$, которые можно переписать в виде С-соотношений $x_{c}^{-1} x_{c-1} x_{c}=x_{c}^{-1}$. Очевидно, теорема 2 является частньм случаем теоремы 3 .

Пусть $G=\langle T \mid R\rangle$ - некоторое конечное копредставление группы $G$. Предположим, что удалось найти $G$-инвариантное подмножество $X$ такое, что соотношения $a^{-1} b a=c^{\varepsilon}(a, b, c \in X, \varepsilon= \pm 1)$ также являются определяюшими соотношениями группы $G$. Из доказательства теоремы 3 для ориентируемого случая [11] нетрудно извлечь следующую процедуру построения конечного задания групшы $G$ с помощью C-соотношений. 
Шаг 1. Считая, что $g \sim g^{\prime} \Longleftrightarrow$ элемент $g$ или $g^{-1}$ сопряжен с $g^{\prime}\left(g, g^{\prime} \in X\right)$, выберем в $X$ множество $B$ представителей классов эквивалентности и перепишем копредставление $G=\langle T \mid R\rangle$ в системе образующих $B$. Это делается стандартным образом. Выразим в группе $G$ элементы множества $B$ через элементы множества $T$, и наоборот

$$
b_{i}=v_{i}\left(t_{1}, \ldots, t_{m}\right), \quad t_{j}=w_{j}\left(b_{1}, \ldots, b_{n}\right) .
$$

Тогда

$$
b_{i}=v_{i}\left(w_{1}\left(b_{1}, \ldots, b_{n}\right), \ldots, w_{m}\left(b_{1}, \ldots, b_{n}\right)\right) .
$$

Кроме того, для каждого определяющего соотношения $r\left(t_{1}, \ldots, t_{m}\right)=1(r \in R)$ имеет место равенство

$$
r\left(w_{1}\left(b_{1}, \ldots, b_{n}\right), \ldots, w_{m}\left(b_{1}, \ldots, b_{n}\right)\right)=1
$$

Соотношения (8) и (9) задают группу $G$ в системе образующих $B$.

Шаг 2. Пусть для элементов $b, c \in B$ слова $u\left(b_{1}, \ldots, b_{m}\right)$ и $\varepsilon= \pm 1$ выполненно равенство $u^{-1} b u=c^{\varepsilon}$. Все такие равенства дают систему определяющих соотношений группы $G$ в системе образующих $B$ (если группа $G_{a b}$ - свободная абелева, то можно ограничиться $\varepsilon=1)$. Выделим конечное множество соотношений вида $u_{i}^{-1} b_{i} u_{i}=c_{i}^{\varepsilon}$, из которых следуют равенства (8) и (9).

Шаг 3. Для каждого слова $u_{i}$, построенного на предыдушем шаге, пусть $u_{i j}-$ его начальньй отрезок длины $j$. Рассмотрим множество $X_{0}$, которое получается добавлением к $B$ элементов $u_{i j}^{-1} b_{i} u_{i j}$. Тогда $X_{0}$ - конечное множество образующих и всевозможные соотношения $a^{-1} b a=c^{\varepsilon}$, где $a, b, c \in X_{0}, \varepsilon= \pm 1$, задают группу $G$.

Следующая теорема дает описание ядра отображения $\sigma: G^{*} \rightarrow G$ для произвольной группы $G$. Напомним, что в предыдущем параграфе был определен гомоморфизм $\rho_{B}: \bigoplus_{g \in B} C(g) \rightarrow H_{2} G$ и разбиение $B=B^{+} \cup B^{-}$, где $B^{+}-$множество ориентируемых, а $B^{-}$- неориентируемых элементов, содержащихся в $B$. Пусть $\mathscr{A}(B)^{+}{ }_{-}$свободная абелева группа со свободными образующими $g \in B^{+}$и $\mathscr{A}(B)^{-}$ - прямое произведение циклических групп порядка 2 с образующими $g \in B^{-}$. Положим $\mathscr{A}(B)=\mathscr{A}(B)^{+} \oplus \mathscr{A}(B)^{-}$и определим гомоморфизм $\tau_{B}: \mathscr{A}(B) \rightarrow H_{1} G$, считая, что

$$
\tau_{B}(g)=g G^{\prime} \in G_{a b} \cong H_{1} G \quad(g \in B)
$$

ТЕОРема 4. Пусть $Y$ - G-инвариантное мнохсество образующих группьи $G, G^{*}=(G, Y)^{*}$ - соответствующее универсальное С-накрытие и В система представителей для отношения әквивалентности (6) на множестве $Y$. Тогда ядро естественного эпиморфизма $\sigma: G^{*} \rightarrow G-$ центральная подгруппа, которую мохсно включить в точную последовательность

$$
\bigoplus_{g \in B} C(g) \stackrel{\rho_{B}}{\longrightarrow} H_{2} G \stackrel{\nu}{\longrightarrow} \operatorname{ker} \sigma \stackrel{\eta}{\longrightarrow} \mathscr{A}(B) \stackrel{\tau_{B}}{\longrightarrow} H_{1} G \longrightarrow 0 .
$$

Гомоморфизмы $\nu$ и $\eta$ будут определены в процессе доказательства. Для ориентируемого С-накрытия $\sigma_{+}: G_{+}^{*} \rightarrow G$ имеет место аналогичная точная последовательность. Надо лишь в качестве $B$ взять множество представителей классов 
сопряженности, содержащихся в $Y$, а в качестве $\mathscr{A}(B)$ - свободную абелеву группу, порожденную элементами $g \in B$.

Как мы уже отмечали, группа $G$ является С-группой тогда и только тогда, когда для некоторого выбора $G$-инвариантного множества образующих ядро отображения $\sigma$ тривиально, поэтому теорема 1 - частный случай теоремы 4 . Доказательство теоремы 4 проведем в три шага.

Шаг 1. Пусть $G$ - нормальное замькание подмножества $B$, которое линейно независимо по модулю коммутанта, и пусть $Y=B^{G}$. Тогда $G^{*} \cong G_{+}^{*}$ и $\operatorname{ker} \sigma \cong$ $\operatorname{coker} \rho_{B}$.

ДокАЗАТЕЛьство. Изоморфизм $G^{*} \cong G_{+}^{*}$ следует из леммы 3. Опишем $\operatorname{ker} \sigma$. Для этого нам понадобятся некоторые факты о центральных расширениях.

Пусть $A$ - тривиальный $G$-модуль. Если группа $G_{a b}$ свободная абелева, то по теореме об универсальных коэффициентах

$$
H^{2}(G, A) \cong \operatorname{Hom}\left(H_{2} G, A\right)
$$

Возьмем $A=H_{2} G$. Тогда в правой части имеется выделенньй элемент тождественное отображение $\mathrm{H}_{2} \mathrm{G} \rightarrow \mathrm{H}_{2} G$. Соответствующий элемент группы $H^{2}\left(G, H_{2} G\right)$ определяет расширение, обладаюшее некоторым универсальным свойством. Опишем это расширение подробнее и установим его связь с С-группами.

Пусть заданы два центральных расширения группы $G$

$$
E_{i}: 1 \longrightarrow A_{i} \longrightarrow \bar{G}_{i} \longrightarrow G \longrightarrow 1 \quad(i=1,2)
$$

Морфизмом $\varphi: E_{1} \rightarrow E_{2}$ расширений назовем гомоморфизм групп $\varphi: \bar{G}_{1} \rightarrow \bar{G}_{2}$, действующий тождественно в факторгрупе $G \cong \bar{G}_{1} / A_{1} \cong \bar{G}_{2} / A_{2}$. Отметим, что, если $\varphi, \varphi^{\prime}: E_{1} \rightarrow E_{2}$ - два морфизма, то для любого элемента $g \in \bar{G}_{1} \varphi^{\prime}(g)=$ $\varphi(g) a_{g}$, где $a_{g}$ лежит в центре. Отсюда следует, что

$$
\varphi^{\prime}([g, h])=\left[\varphi(g) a_{g}, \varphi(h) a_{h}\right]=\varphi([g, h]) \quad\left(g, h \in \bar{G}_{1}\right),
$$

т.е. любые два морфизма совпадают на коммутанте.

Центральное расширение $\widehat{E}$ групшы $G$ назовем универсальным центральным расширением, если для любого центрального расширения $E$ сушествует морфизм $\varphi: \widehat{E} \rightarrow E$, который определен однозначно с точностью до единственного морфизма $\alpha: E \rightarrow E$, действуюшего тождественно на ядре расширения $E$.

Понятно, что, если универсальное центральное расширение сушествует, то оно единственно с точностью до изоморфизма, тождественного в факторгруппе $G$.

ПрЕДЛОЖЕНИЕ 1. Если $G_{a b}-$ свободная абелева группа, то универсальное иентральное расширение $\widehat{E}$ существует. Его ядро изоморфно $H_{2} G$, m.е. $\widehat{E}$ имеет вид

$$
\widehat{E}: \quad 0 \longrightarrow \mathrm{H}_{2} G \longrightarrow \widehat{G} \longrightarrow G \longrightarrow 1 \text {. }
$$


Эпиморфизм $\widehat{G} \rightarrow G$ индуцирует изоморфизм факторгрупп по коммутанту $\widehat{G}_{a b} \cong G_{a b}$.

В литературе часто встречается частный случай этого утверждения, когда группа $G$ совершенна, т.е. $G=G^{\prime}$. Тогда сушествует единственный морфизм $\varphi: \widehat{E} \rightarrow E$ и $H_{2} \widehat{G}=0$ (см., например, [12]). Универсальное центральное расширение используется также для конечных групп [13], но тогда оно определено неоднозначно. По этой причине мы все же приведем доказательство предложения 1, хотя оно достаточно стандартно.

ДокАЗАТЕЛЬство. Представим группу $G$ в виде факторгруппы свободной групшы: $G=F / N$. Пусть $\widetilde{G}=F /[N, F], \widetilde{N}=N /[N, F]$. Для центральной подгрупшы $\widetilde{N}$ можно написать разложение $\widetilde{N} \cong H_{2} G \times D$, где $D$ отображается изоморфно на подгруппу свободной абелевой группы $\widetilde{G}_{a b} \cong F_{a b}$. Факторизуя $\widetilde{G}_{a b}$ по образу $D$, мы получим, очевидно, $G_{a b}$, а так как по условию $G_{a b}-$ свободная абелева групп, то можно написать разложение $\widetilde{G}_{a b} \cong G_{a b} \times D$. Таким образом, определен эпиморфизм $\widetilde{G} \rightarrow D$. Обозначим через $\widehat{G}$ ядро этого эпиморфизма. Непосредственно из построения следует, что имеет место точная последовательность (10) и что $\widehat{G}_{a b} \cong G_{a b}$. Проверим универсальное свойство построенного расширения. Для этого рассмотрим произвольное центральное расширение групшы $G$

$$
E: \quad 1 \longrightarrow A \longrightarrow G \longrightarrow G
$$

Пусть $x_{i}$ - свободные образуюшие группы $F$ и $h_{i}-$ элементы группы $\bar{G}$, имеющие те же образы в группе $G$. Тогда отображение $x_{i} \rightarrow h_{i}$ индуцирует гомоморфизм $\widetilde{G} \rightarrow \bar{G}$, ограничение которого на $\widehat{G}$ дает нужный морфизм $\varphi: \widehat{E} \rightarrow E$. Предположим, что имеется два морфизма $\varphi, \varphi^{\prime}: \widehat{E} \rightarrow E$, и пусть $\varphi^{\prime}=\alpha \varphi$, где $\alpha: E \rightarrow E$ действует тождественно на $A$. Очевидно $\alpha$ определяется своими значениями на системе представителей смежных классов $\bar{G}$ по модулю $A$. Пусть $\widehat{g}-$ система представителей в $\widehat{G}$. Рассмотрим систему представителей $\bar{g}=\varphi(\widehat{g})$ в $\bar{G}$. Тогда $\alpha(\bar{g})=\bar{g} a_{g}$, где $a_{g} \in A$ и

$$
a_{g}=\bar{g}^{-1} \alpha(\bar{g})=\varphi(\widehat{g})^{-1} \alpha(\varphi(\widehat{g}))=\varphi(\widehat{g})^{-1} \varphi^{\prime}(\widehat{g})
$$

Отсюда следует, что, если автоморфизм $\alpha$ существует, то он определен однозначно. Теперь ясно, как построить $\alpha$ : положим по определению

$$
\alpha(\bar{g} a)=\bar{g} a_{g} a, \quad \text { где } a_{g}=\varphi(\widehat{g})^{-1} \varphi^{\prime}(\widehat{g}) \quad(a \in A) .
$$

Для $c \in H_{2} G \subseteq \widehat{G}$ имеем

$$
\alpha(\varphi(\widehat{g} c))=\alpha(\bar{g} \varphi(c))=\bar{g} a_{g} \varphi(c)=\bar{g} \varphi(\widehat{g})^{-1} \varphi^{\prime}(\widehat{g}) \varphi(c)=\varphi^{\prime}(\widehat{g}) \varphi(c)
$$

Как мы уже отмечали, любые два морфизма совпадают на коммутанте, а так как $\widehat{G}^{\prime} \supseteq H_{2} G$, то $\varphi(c)=\varphi^{\prime}(c)$, следовательно $\alpha(\varphi(\widehat{g} c))=\varphi^{\prime}(\widehat{g} c)$. Легко также проверить, что $\alpha$ - гомоморфизм. 
ЛЕмма 5. Пусть $\widehat{G}$ - универсальное иентральное расширение группь $G$. Если образ $Y$ множества $X \subseteq \widehat{G}$ порожсдает группу $G$, то множество $X$ nорохсдает группу $\widehat{G}$.

Действительно, пусть $\bar{G}$ - подгруппа, порожденная множеством $X$ и $A=\bar{G} \cap$ $H_{2} G \subseteq \widehat{G}$. Имеет место точная последовательность $(11)$ и вложение $i: \bar{G} \rightarrow \widehat{G}$ является морфизмом расширений. С другой стороны, в силу универсального свойства расширения $\widehat{G}$ сушествует гомоморфизм $\varphi: \widehat{G} \rightarrow \bar{G}$. Композиция $\varphi$ с вложением - это некоторый морфизм $\widehat{E} \rightarrow \widehat{E}$. По свойству единственности он отличается от тождественного на морфизм, оставляющий неподвижными элементы из $\mathrm{H}_{2} G$. В частности, $i \varphi-$ эпиморфизм, следовательно, $i$ - изоморфизм.

СЛЕДСТВИЕ 3. Пусть әруппа $G$ совпадает с нормальным замыканием множсества $B$, и пусть $\widehat{B}$-мнохсество представителей элементов из $В$ в универсальном чентральном расширении $\widehat{G}$. Тогда $\widehat{G}$ совпадает с нормальныц замыканием множества $\widehat{B}$.

Лемма 6. Предположим, что группа $G$ совпадает с нормальным замыканием множества әлементов $B$, линейно независимых в $G_{a b}$. Тогда универсальное иентральное расширение $\widehat{G}$ группь $G$ является С-группой.

ДоказАтЕльство. Положим $Y=B^{G}, X=\widehat{B}^{\widehat{G}}$, где $\widehat{B}$ - множество представителей элементов $g \in B$. Рассмотрим С-групшы $G^{*}=(G, Y)^{*}$ и $\widehat{G}^{*}=(\widehat{G}, X)^{*}$. Тогда имеет место коммутативная диаграмма

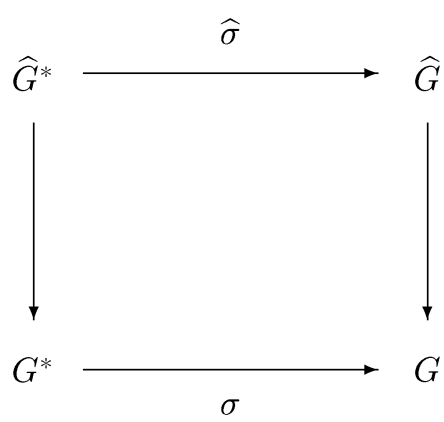

По лемме 2 прообраз центральной подгруппы $H_{2} G \subseteq \widehat{G}$ в универсальном С-накрытии $\widehat{G}^{*}$ лежит в центре, поэтому $\widehat{G}^{*}$ - центральное расширение групшы $G$. По универсальному свойству расширения $\widehat{G}$ сушествует морфизм $\varphi: \widehat{G} \rightarrow \widehat{G}^{*}$. В силу единственности композиция $\varphi$ с $\widehat{\sigma}$ с точностью до морфизма $\alpha: \widehat{G} \rightarrow \widehat{G}$, оставляюшего неподвижными элементы из $H_{2} G$, совпадает с тождественным отображением. Отсюда следует, что $\widehat{G}^{*} \cong \widehat{G} \times K$, где $K \cong \operatorname{ker} \widehat{\sigma}$. Так как $K$ - центральная подгруппа, то, переходя к факторгруппе по коммутанту, получим $\widehat{G}_{a b}^{*} \cong \widehat{G}_{a b} \times K$. Но из условия о линейной независимости множества $B$ следует, что $\widehat{\sigma}$ индуцирует изоморфизм $\widehat{G}^{*} \cong \widehat{G}$, поэтому прямое слагаемое $K$ должно быть тривиально.

Теперь нетрудно доказать утверждение, выделенное как шаг 1. Мы только что доказали, что $\widehat{G} \cong(\widehat{G}, X)^{*}$, где $X=\widehat{B}^{G}$. По универсальному свойству расширения $\widehat{G}$ сушествует морфизм $\varphi: \widehat{G} \rightarrow G^{*}$, который очевидно является отображением "на". Определим гомоморфизм $\nu$ в точной последовательности теоремы 4 как 
ограничение $\varphi$ на $H_{2} G$. Поскольку $H_{2} G$ содержится в коммутанте, $\nu$ не зависит от выбора $\varphi$. Если $g \in B$ и $h \in C(g)$, то $\nu\left(\rho_{B}(h)\right)=\nu(g \wedge h)=[g, h]=1$, поэтому определен индуцированный эпиморфизм $\psi: \widetilde{G} \rightarrow G^{*}$, где $\widetilde{G} \cong \widehat{G} / \operatorname{Im} \rho_{B}$. Нам надо доказать, что $\psi$ - изоморфизм. Пусть $\widehat{g} \in \widehat{B}$ - прообраз элемента $g \in B$. Группа $\widetilde{G}$ получается из $\widehat{G}$ добавлением соотношений $\widehat{g}=\widehat{g}^{h}(g \in B, h \in C(g))$. Так как $\widehat{g}, \widehat{g}^{h} \in X$, эти соотношения, введенные в группе $\widehat{G}=(\widehat{G}, X)^{*}$, определяют С-группу $(\widetilde{G}, T)^{*}(T-$ образ $X$ в $\widetilde{G})$. Гомоморфизм универсальных С-накрытий

$$
\widetilde{G} \cong(\widetilde{G}, T)^{*} \longrightarrow G^{*}=(G, Y)^{*}
$$

является изоморфизмом тогда и только тогда, когда соответствуюшее отображение $T \rightarrow Y$ биективно. Группа $G$ действует на множествах $Y$ и $T$. Из линейной независимости элементов множества $B$ по модулю коммутанта следует, что $Y$ объединение орбит вида $g^{G}$, где $g \in B$, а $T$ - объединение орбит вида $\widetilde{g}^{G}$, где $\widetilde{g}$ образ $\widehat{g} \in \widehat{B}$. На каждой орбите отображение $\widetilde{g}^{G} \rightarrow g^{G}$ биективно, так как, если $h \in C(g)$, то и $\widetilde{g}^{h}=\widetilde{g}$. На этом заканчивается рассмотрение шага 1.

Шаг 2. Рассмотрим теперь ориентируемое С-накрытие. Пусть $(G, Y)$ - некоторая пред-С-группа и $B$ - множество представителей классов сопряженности, содержащихся в $Y$. Рассмотрим точную последовательность абелевых групп

$$
1 \longrightarrow A \longrightarrow \mathscr{A}(B) \stackrel{\tau_{B}}{\longrightarrow} G_{a b} \longrightarrow 1,
$$

где $\mathscr{A}(B)$ - свободная абелева группа с базисом $B, A=\operatorname{ker} \tau_{B}$. Пусть $\bar{G}$ - подгруппа прямого произведения $G \times \mathscr{A}(B)$, состоящая из пар $(g, a)$ таких, что образы элементов $g$ и $a$ в $G_{a b}$ совпадают. Отображение $a \rightarrow(1, a)(a \in A)$ определяет вложение $A \rightarrow \bar{G}$ и $\bar{G} / A \cong G$, т.е. $\bar{G}$ - центральное расширение группы $G$. Oчевидно, $\bar{G}^{\prime} \cong G^{\prime}$ и $\bar{G}_{a b} \cong \mathscr{A}(B)$. Положим $\bar{B}=\{\bar{g} \in \bar{G} \mid \bar{g}=(g, g)(g \in B)\}$, $\bar{Y}=\bar{B}^{\bar{G}}$. Естественное отображение $\bar{G} \rightarrow G$ определяет биекцию $\bar{Y} \rightarrow Y$, поэтому ориентируемые С-накрытия $G^{*}=(G, Y)^{*}$ и $\bar{G}^{*}=(\bar{G}, \bar{Y})^{*}$ изоморфны. Отсюда следует, что ядра соответствуюших накрываюших отображений $\sigma, \bar{\sigma}$ можно включить в точную последовательность

$$
1 \longrightarrow \operatorname{ker} \bar{\sigma} \longrightarrow \operatorname{ker} \sigma \longrightarrow A \rightarrow 1 .
$$

Используя шаг 1 и точную последовательность (12), имеем

$$
\bigoplus_{\bar{g} \in \bar{B}} C(\bar{g}) \stackrel{\rho_{\bar{B}}}{\longrightarrow} H_{2} \bar{G} \longrightarrow \operatorname{ker} \sigma \longrightarrow \mathcal{A}(B) \longrightarrow H_{1} G \longrightarrow 0
$$

Осталось показать, что coker $\rho_{\bar{B}} \cong \operatorname{coker} \rho_{B}$. Рассмотрим коммутативную диаграмму

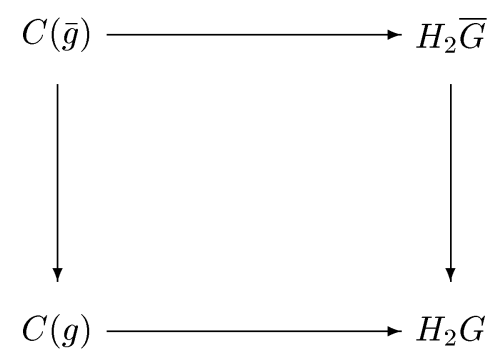


Нам достаточно доказать, что вертикальные стрелки - эпиморфизмы. Для правой это следует из пятичленной точной последовательности

$$
\mathrm{H}_{2} \overline{\mathrm{G}} \longrightarrow \mathrm{H}_{2} \mathrm{G} \longrightarrow \bar{G}_{a b} \longrightarrow G_{a b} \longrightarrow 1 .
$$

Для отображения слева это очевидно из определения $\bar{G}$.

Шаг 3. Рассмотрим обший случай. Предположим, что $B$ - множество представителей классов сопряженности, содержашихся в $Y$. Можно предполагать, что, если $g \in B, g^{-1} \in Y$ и элемент $g$ ориентируем, то $g^{-1} \in B$. На шаге 2 ядро ker $\sigma_{+}$ ориентируемого $\mathrm{C}$-накрытия было описано с помощью соответствующей точной последовательности. Чтобы получить $G^{*}$ из $G_{+}^{*}$ нужно профакторизовать по элементам $x_{c} x_{c-1}\left(c, c^{-1} \in Y\right)$, причем можно ограничиться такими соотношениями, для которых $c \in B$. Если $c$ ориентируем, то образ $x_{c} x_{c^{-1}}$ в $\mathscr{A}(B)-$ это $c-c^{-1}$, а соотношение $c=c^{-1}$ означает, что мы удаляем из базы $\mathscr{A}(B)$ один из элементов $c, c^{-1}$. Если $c$ неориентируем, то элементу $x_{c} x_{c^{-1}}$ соответствует $2 c$. Отсюда очевидно, что для описания $\operatorname{ker} \sigma$ (вместо $\operatorname{ker} \sigma_{+}$) необходимо определить $\mathscr{A}(B)$ именно так, как мы сделали в теореме 4 . Это заканчивает доказательство.

\section{§ 3. Неприводимые ориентируемые С-группы}

Частным случаем теоремы 1 является следующее утверждение.

СлеДСТВИЕ 4. Группа $G$ - неприводимая ориентируемая С-группа тогда и только тогда, когда $G$ совпадает с нормальным замыканием некоторого әлемента $g \in G, G_{a b}$ - бесконечная ииклическая группа и любой әлемент зруппь $\mathrm{H}_{2} G$ имеет вид $g \wedge h(h \in C(g))$.

Мы покажем, как можно использовать это утверждение в конкретных случаях. Начнем с нескольких лемм, облегчающих проверку условий следствия.

Если группа $G$ совпадает с нормальным замыканием элемента $g \in G$, то, очевидно, $G_{a b}$ - циклическая группа с образуюшим $g G^{\prime}$. Для разрешимых групп верно и обратное.

Лемма 7. Пусть $G$-разрешимая группа. Тогда $G$ совпадает с нормальным замыканием әлемента $g$ в том и только том случае, когда $G_{a b}-$ ииклическая группа с образующим $g G^{\prime}$.

ДоказАтельство. Лемма очевидна, если $G$ - абелева группа. Пусть $G$ имеет ступень разрешимости $l>1$, и пусть $A=G^{(l-1)}$ - последний нетривиальный член ряда коммутантов. Если $R$ - нормальное замыкание элемента $g$, то по индукции $G=R A$, поэтому

$$
G / R=R A / R=A / A \cap R
$$

- абелева группа, следовательно, $G^{\prime} \subseteq R$. Так как $G$ порождается по модулю $G^{\prime}$ элементом $g$, отсюда следует, что $G=R$.

Отметим, что в общем случае нет ничего похожего на лемму 7. Пусть, например, $H$ - произвольная группа без кручения, совпадаюшая со своим коммутантом, и пусть $G=\langle g\rangle * H$ - ее свободное произведение с бесконечной циклической группой. Тогда $G_{a b} \cong\langle g\rangle$, однако, как доказал Клячко [14], $G$ не совпадает с нормальным замыканием никакого своего элемента. 
Лемма 8. Пусть $G_{a b}-$ ииклическая группа с образующим $g G^{\prime}$. Тогда әлемент $g-1 \in \mathbb{Z}\langle g\rangle$ действует сюргективно в $G^{\prime} / G^{\prime \prime}$. Если к тому же группа $G$ конечно порохсдена, то действие $g-1$ в $G^{\prime} / G^{\prime \prime}$ биективно.

ДоказАтельство. Очевидно, можно предполагать, что $G^{\prime \prime}=1$. Тогда для любых $a, b \in G^{\prime}$

$$
\left[g^{n} a, g^{m} b\right]=\left[g^{n}, b\right]\left[a, g^{m}\right]=b^{1-g^{n}} a^{g^{m}-1}=c^{g-1}
$$

где $c \in G^{\prime}$. Отсюда следует, что $G^{\prime}$ состоит из элементов вида $c^{g-1}\left(c \in G^{\prime}\right)$. Если группа $G$ конечно порождена, то $G^{\prime}$ - конечно порожденный модуль над нетеровьм кольцом $\mathbb{Z}\langle g\rangle$. Пусть $f$ - эндоморфизм этого модуля, определенный действием $g-1$. Если ker $f \neq 0$, то, поскольку $f$ - эпиморфизм, цепочка подмодулей

$$
\operatorname{ker} f \subseteq \operatorname{ker} f^{2} \subseteq \cdots \subseteq \operatorname{ker} f^{n} \subseteq \cdots
$$

строго возрастает. Это противоречит нетеровости модуля $G^{\prime}$.

ПримеР 1. Если $G$ - неприводимая С-группа, то $G^{\prime} / G^{\prime \prime}$ не может быть циклической группой четного порядка.

Действительно, если $G^{\prime} / G^{\prime \prime}$ - циклическая группа четного порядка с образуюшим $a$, то $a^{g}=a^{k}$, где $k \in \mathbb{Z}, k$ нечетно. Тогда $g^{g-1}=a^{k-1}$, но возведение в четную степень не будет отображением "на".

Лемма 9. Пусть группа $G$ конечно порождена и $G_{a b}$ - бесконечная ииклическая группа. Тогда вложсение $G^{\prime} \rightarrow G$ индуцирует изоморфизм $H_{2} G^{\prime} \otimes_{G} \mathbb{Z} \cong H_{2} G$.

ДокАЗАТЕЛьство. Из спектральной последовательности расширения

$$
1 \longrightarrow G^{\prime} \longrightarrow G \longrightarrow G_{a b} \longrightarrow 1
$$

очевидно следует, что имеет место точная последовательность

$$
0 \longrightarrow H_{2} G^{\prime} \otimes_{G} \mathbb{Z} \longrightarrow H_{2} G \longrightarrow H_{1}\left(G_{a b}, H_{1} G^{\prime}\right) \longrightarrow 0
$$

Пусть $g$ - образующий группы $G_{a b}$. Ее гомологии можно вычислить с помощью резольвенты

$$
0 \longrightarrow \mathbb{Z}\langle g\rangle \stackrel{g-1}{\longrightarrow} \mathbb{Z}\langle g\rangle \longrightarrow 0 .
$$

По лемме $8 g-1$ действует биективно в $G^{\prime} / G^{\prime \prime}$, поэтому $H_{1}\left(G_{a b}, H_{1} G^{\prime}\right)=0$.

ЗАмЕчАНИЕ. Используя леммы 7-9 полезно иметь ввиду, что, если $N \triangleleft G, G / N$ - циклическая группа с образуюшим $g N$ и $g-1$ действует сюръективно в $N_{a b}$, то $N=G^{\prime}$. Действительно, так как $N \supseteq G^{\prime} \supseteq N^{\prime}$, можно предполагать, что группа $N$ абелева. Тогда, по условию, для любого $a \in N$ существует элемент $c \in N$ такой, что $a=c^{g-1}=[c, g] \in G^{\prime}$, откуда $N=G^{\prime}$. 
ПримеР 2. Пусть $Q$ - группа кватернионов

$$
Q=\left\langle a, b, c \mid a^{2}=b^{2}=c, c^{2}=1, c=[a, b], a c=c a, b c=c b\right\rangle .
$$

Легко видеть, что отображение $a \rightarrow b, b \rightarrow a b, c \rightarrow c$ продолжается до автоморфизма группы $Q$. Рассмотрим полупрямое произведение $G=\langle g\rangle Q$, в котором $g$ действует на $Q$ как автоморфизм, определенньй вьше. Очевидно, $Q_{a b}$ - элементарная 2-группа, порожденная элементами $\widetilde{a}=a Q^{\prime}$ и $\widetilde{b}=b Q^{\prime}$. Элемент $g-1$ действует в $Q_{a b}$ следуюшим образом

$$
\widetilde{a} \rightarrow \widetilde{a} \widetilde{b}, \quad \widetilde{b} \rightarrow \widetilde{a},
$$

откуда следует, что $g-1$ - автоморфизм группы $Q_{a b}$. В соответствии с замечанием после леммы 9 имеем $Q=G^{\prime}$. Так как группа $G$ разрешима, то по лемме 7 она совпадает с нормальньм замыканием элемента $g$. Как хорошо известно, $H_{2} Q=0$ (это, например, следует из периодичности когомологий Тейта группы $Q$ ). По лемме $9 H_{2} G=0$. Применяя следствие 4 , мы видим, что $G$ - неприводимая ориентируемая С-группа. Нетрудно задать группу $G$ как С-группу. Для этого рассмотрим класс сопряженности элемента $g$. Он содержит четыре элемента

$$
g, \quad x=g a, \quad y=g b, \quad z=g a b .
$$

Группа $G$ задается образующими $g, x, y, z$ и соотношениями сопряженности, которые выполнены между ними в $G$. Таких соотношений, очевидно, 12 . Несложные вычисления показывают, что достаточно шести соотношений

$$
\begin{aligned}
& g^{-1} x g=y, \quad g^{-1} y g=z, \quad g^{-1} z g=x, \\
& y^{-1} g y=x, \quad y^{-1} x y=z, \quad y^{-1} z y=g .
\end{aligned}
$$

Неприводимые С-группы естественно изучать в зависимости от свойств коммутанта. Рассмотрим простейший случай, когда коммутант абелев.

Теорема 5. Пусть $G$ - конечно породжденная метабелева группа. Тогда следующие условия әквивалентнь:

(i) $G$ - неприводимая ориентируемая $\mathrm{C}-$-рупппа;

(ii) $G_{a b}$ - бесконечная циклическая группа и $\mathrm{H}_{2} G=0$;

(iii) $G=\langle g\rangle M-$ полупрямое произведение бесконечной циклической группь $\langle g\rangle$ и абелева нормального делителя $M$, причем $g-1$ действует сюрвективно в $M$ и $M \wedge M$.

Отметим, что в формулировке этой теоремы имеется в виду, что $g$ действует во внешнем произведении $M \wedge M$ диагонально, т.е.

$$
\left(m_{1} \wedge m_{2}\right)(g-1)=m_{1} g \wedge m_{2} g-m_{1} \wedge m_{2} \quad\left(m_{1}, m_{2} \in M\right) .
$$


ДОКАЗАТЕЛЬСТВО ТЕОРЕМЫ 5. Пусть $G$ - конечно порожденная метабелева группа. Если $G$ - неприводимая С-группа, то по следствию $4 G$ совпадает с нормальным замыканием некоторого элемента $g \in G$. Чтобы доказать импликацию (i) $\Longrightarrow$ (ii), нам достаточно проверить, что $H_{2} G=0$. По лемме $8 g-1$ действует биективно в $G^{\prime}$, следовательно, если $a \neq 1, a \in G$, то $[a, g]=a^{g-1} \neq 1$. Это означает, что $C(g)=\langle g\rangle$. Так как $g \wedge g=0$, то, применяя следствие 4 , получим $\mathrm{H}_{2} G=0$. Импликация (ii) $\Longrightarrow(\mathrm{i})$ вытекает из леммы 7 и следствия 4. Проверим, что (ii) $\Longrightarrow$ (iii). Если вьполнено условие (ii), то, очевидно, $G=\langle g\rangle M$, где $M=G^{\prime}$ и по лемме $8 g-1$ действует на $M$ биективно. По лемме 9

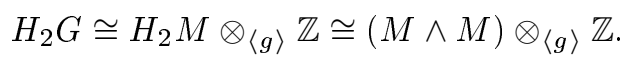

Так как $H_{2} G=0$, это означает сюръективность действия $g-1$ на $M \wedge M$. Так же легко получается импликация (iii) $\Longrightarrow$ (ii).

ПримеР 3. Пусть $G=\langle g\rangle M$ - полупрямое произведение бесконечной циклической группы и свободной абелевой группы конечного ранга. Тогда $M \wedge M$ - также свободная абелева группа конечного ранга. Группа $G$ будет неприводимой (конечно определенной) С-группой в том и только том случае, когда $g-1$ индуцирует на $M$ и $M \wedge M-$ эндоморфизмы с определителем \pm 1 .

ПримеР 4 . Пусть $M$ - конечномерное пространство над простым полем $\mathbb{Z}_{p}$, и пусть $f$ - невырожденное линейное преобразование пространства $M$. Рассмотрим полупрямое произведение $G=\langle g\rangle M$, в котором $g$ действует на $M$, как линейное преобразование $f$. Группа $G$ будет неприводимой (конечно определенной) С-группой в том и только том случае, когда $g-1$ индуцирует на $M$ и $M \wedge M-$ невырожденные линейные преобразования. Если $\lambda_{i}$ - собственные значения преобразования $f$ в алгебраическом замыкании поля $\mathbb{Z}_{p}$, то это означает, что $\lambda_{i} \neq 0 ; 1$ и $\lambda_{i} \lambda_{j} \neq 1$ при $i \neq j$.

В качестве множества С-образуюших неприводимой С-групшы $G$ с абелевым коммутантом можно взять класс сопряженности элемента $g \in G$ такого, что $g G^{\prime}-$ образующий группы $G_{a b}$. Легко видеть, что этот класс сопряженности совпадает с классом смежности $g G^{\prime}$.

Другие примеры С-групп с абелевым коммутантом можно найти в работе [11]. Там же указан пример неприводимой ориентируемой С-группы $G$ такой, что $G^{\prime \prime}$ лежит в центре, но $H_{2} G \neq 0$.

Рассмотрим противоположный случай, когда коммутант $G^{\prime}$ неприводимой С-группы $G$ близок к простой группе.

Лемма 10. Пусть $N$ - простая группа и $\widehat{N}$ - ее универсальное центральное расиирение. Тогда прямое произведение $G=\langle t\rangle \times \widehat{N}$ является неприводимой ориентируемой $\mathrm{C}-$-руппой.

Действительно, пусть $x \in N, x \neq 1$. Так как $N$-простая группа, то нормальное замыкание элемента $x$ совпадает с $N$. Тогда по следствию 3 нормальное замыкание любого его прообраза $y \in \widehat{N}$ совпадает с $\widehat{N}$. Отсюда следует, что $\langle t\rangle \times \widehat{N}=G$ - нормальное замькание элемента $t y$. Остается заметить, что $H_{2} G=H_{2} \widehat{N}=0$. В качестве множества С-образующих группы $G$ можно взять класс сопряженности элемента $g=t y$, где $y$ - любой нецентральный элемент групп $\widehat{N}$. 
ПримеР 5 . Пусть $N=P S L(n, q)$ - проективная специальная линейная группа над полем из $q$ элементов. Если $(n, q) \neq(2,2),(2,3)$, то, как известно, $N$ - простая группа. Если к тому же $(n, q) \neq(2,4),(2,9),(3,2),(3,4),(4,2)$, то универсальное центральное расширение $\widehat{N}$ изоморфно специальной линейной группе $S L(n, q)$ (см. [13, табл. 4.1]). По лемме $4 t \times S L(n, q)$-неприводимая ориентируемая группа.

Во всех предыдущих примерах вторая группа гомологий была тривиальна. Приведем пример, в котором следствие 4 работает в полном объеме.

ПримеР 6. Прямое произведение $G=\langle t\rangle \times P S L(n, q)$ за исключением семи случаев, перечисленных в примере 5 , является неприводимой $\mathrm{C}$-группой.

Для доказательства рассмотрим образ $x$ в группе $P S L(n, q)=N$ следуюшей матрицы $y \in S L(n, q)=\widehat{N}$

$$
\left(\begin{array}{ccccc}
0 & 0 & \ldots & 0 & (-1)^{n-1} \\
1 & 0 & \ldots & 0 & 0 \\
0 & 1 & \ldots & 0 & 0 \\
\ldots & \ldots & \ldots & \ldots & \ldots \\
0 & 0 & \ldots & 1 & 0
\end{array}\right)
$$

Так как группа $N$ проста, $G$ совпадает с нормальным замыканием элемента $g=t x$. Проверим, что группа $H_{2} G$ состоит из элементов $g \wedge h$, где $h \in C(g)$. Очевидно, $H_{2} G \cong H_{2} N$ и $C_{G}(g)=t \times C_{N}(x)$. Так как для $z \in C_{N}(x) t x \wedge t z=x \wedge z$, то нам достаточно проверить, что $H_{2} N$ исчерпьвается элементами $x \wedge z$. Группа $H_{2} N$ естественно отождествляется с ядром отображения $\widehat{N} \rightarrow N$, т.е. с группой скалярных матриц $a E$ с определителем 1 . Пусть $z \in C_{N}(x)$ и $w$-матрица, являющаяся прообразом элемента $z$ в группе $\widehat{N}$. Тогда $x \wedge z=[y, w]$. Надо доказать, что, меняя $z$, мы получим все скалярные матрицы с определителем 1 . Пусть $F_{q}-$ основное поле. Достаточно найти матрицу $w$ такую, что $[y, w]=a E$, где $a$ - первообразный корень из 1 степени $d=(n, q-1)$. Рассмотрим эквивалентное равенство

$$
y w=a w y .
$$

Возьмем в качестве $w$ диагональную матрицу с элементами $1, a, \ldots, a^{n-1}$ по диагонали. Легко проверить, что тогда равенство (13) выполнено. Если $n$ нечетно, то $\operatorname{det}(w)=a^{n(n-1) / 2}=1$ и $w$ - искомая матрица. То же самое верно, если характеристика поля равна 2 . Пусть $n$ четно, $q$ - нечетно, и $m=n / d$. Если $m$ четно, то $\operatorname{det}(w)=\left(a^{n / 2}\right)^{n-1}=\left(a^{d}\right)^{(m / 2)(n-1)}=1$, и снова диагональная матрица дает решение. Предположим, что $m$ нечетно. Тогда $\operatorname{det}(w)=\left(a^{n / 2}\right)^{(n-1)}=$ $\left(a^{d / 2}\right)^{m(n-1)}=-1$. Пусть $l=(q-1) / d$. Если $l$ четно, то $a=b^{2}$, где $b \in F_{q}$. Заменяя $w$ на $b w$, мы не нарушим равенство (13), но теперь уже $\operatorname{det}(b w)=-b^{n}=$ $-a^{(n / 2)}=1$. Рассмотрим случай, когда $l$ нечетно, т.е. $a$ не является квадратом в $F_{q}$. 
Легко проверить, что любая матрица вида

$$
c=\left(\begin{array}{cccccc}
c_{0} & c_{1} & c_{2} & c_{3} & \ldots & c_{n-1} \\
-c_{n-1} & c_{0} & c_{1} & c_{2} & \ldots & c_{n-2} \\
-c_{n-2} & -c_{n-1} & c_{0} & c_{1} & \ldots & c_{n-3} \\
\ldots \ldots \ldots \ldots \ldots \ldots \ldots & \ldots \ldots & \ldots \ldots \ldots \ldots \\
-c_{1} & -c_{2} & \ldots & & -c_{n-1} & c_{0}
\end{array}\right)
$$

где $c_{0}, c_{1}, \ldots, c_{n-1} \in F_{q}$, перестановочна с $y$, поэтому, заменяя $w$ на $w c$, мы не нарушим равенство (13). Если доказать, что существуют элементы $c_{0}, c_{1}, \ldots, c_{n-1} \in F_{q}$ такие, что $\operatorname{det}(c)=-1$, то $w c$ будет искомой матрицей. Доказательство распадается на два случая. Предположим сначала, что $m=1$, т.е. что $n=d$ делит $q-1$. Так как $a$ не является квадратом в поле $F_{q}$, квадратичное расширение $F_{q^{2}}$ состоит из элементов вида $\alpha+\beta b$, где $\alpha, \beta \in F_{q}, b^{2}=a$. Поскольку $a$ - первообразный корень степени $n$ из 1 , поле $F_{q^{2}}$ содержит все корни $n$-й степени из -1

$$
b_{1}=b, \quad b_{2}=b a, \ldots, b_{n}=b a^{n-1}
$$

Непосредственно проверяется, что векторы

$$
u_{k}=\left(1, b_{k}, b_{k}^{2}, \ldots, b_{k}^{n-1}\right) \quad(k=1, \ldots, n)
$$

- собственные векторы матрицы $c$, соответствуюшие собственным значениям

$$
\lambda_{k}=c_{0}+c_{1} b_{k}+\cdots+c_{n-1} b_{k}^{n-1}
$$

Так как эти векторы линейно независимы, отсюда следует, что матрища $c$ диагонализируема над $F_{q^{2}}$, поэтому

$$
\operatorname{det}(c)=\prod_{k=1}^{n}\left(c_{0}+c_{1} b_{k}+\cdots+c_{n-1} b_{k}^{n-1}\right)
$$

Вектор $u_{k}$ можно представить в виде $u_{k}=v_{k}+b_{k} w_{k}$, где координаты векторов

$$
\begin{aligned}
v_{k} & =\left(c_{0}, 0, c_{2} b_{k}^{2}, 0, \ldots, c_{n-2} b_{k}^{n-2}, 0\right), \\
w_{k} & =\left(0, c_{1}, 0, c_{3} b_{k}^{2}, \ldots, 0, c_{n-1} b_{k}^{n-2}\right)
\end{aligned}
$$

уже принадлежат полю $F_{q}$. Корни четной степени $n$ из -1 встречаются парами $\pm b_{k}$. Каждой такой паре соответствует двумерное над $F_{q}$ инвариантное подпространство, порожденное векторами $v_{k}, w_{k}$. Векторы $v_{1}, w_{1}, \ldots, v_{n / 2}, w_{n / 2}$ образуют базис, в котором матрица $c$ распадается на блоки размера $2 \times 2$

$$
\left(\begin{array}{cc}
\alpha_{k} & \beta_{k} \\
b_{k}^{2} \beta_{k} & \alpha_{k}
\end{array}\right)
$$


Здесь $\alpha_{k}+b_{k} \beta_{k}=\lambda_{k}$, т.е.

$$
\begin{aligned}
\alpha_{k} & =c_{0}+c_{2} b_{k}^{2}+\cdots+c_{n-2} b_{k}^{n-2}, \\
\beta_{k} & =c_{1}+c_{3} b_{3}^{2}+\cdots+c_{n-1} b_{k}^{n-2} .
\end{aligned}
$$

Отсюда получается выражение для определителя, использующее только элементы поля $F_{q}$,

$$
\operatorname{det}(c)=\prod_{k=1}^{n / 2}\left(\alpha_{k}^{2}-b_{k}^{2} \beta_{k}^{2}\right) .
$$

Легко видеть, что матрица коэффициентов, с помощью которых элементы $\alpha_{1}, \beta_{1}$, $\ldots, \alpha_{n / 2}, \beta_{n / 2}$ выражаются через $c_{0}, \ldots, c_{n-1}$, невырождена, поэтому эти элементы независимо пробегают поле $F_{q}$. Доказательство (в случае $m=1$ ) будет закончено, если мы покажем, что для любого фиксированного ненулевого элемента $e \in F_{q}$ квадратичная форма $x_{1}^{2}+e x_{2}^{2}$ принимает в $F_{q}$ все значения. Беря $x_{2}=0$, получим все квадраты поля $F_{q}$. Если $e$ не является квадратом в $F_{q}$, то при $x_{1}=0$ получаются все элементы из $F_{q}^{*} \backslash\left(F_{q}^{*}\right)^{2}$. Пусть $e \in\left(F_{q}^{*}\right)^{2}$. После очевидной замены дело сводится к форме $x_{1}^{2}+x_{2}^{2}$. Если для всех $x_{1}, x_{2} \in F_{q} x_{1}^{2}+x_{2}^{2}-$ квадрат в поле $F_{q}$, то множество всех квадратов (вместе с нулем) образуют подполе, содержашее $(q+1) / 2$ элементов. Это невозможно, так как $q$ и $(q+1) / 2$ взаимно просты. Итак, $x_{1}^{2}+x_{2}^{2} \notin\left(F_{q}^{*}\right)^{2}$ для некоторой пары $\left(x_{1}, x_{2}\right)$. Пропорциональные пары дадут все остальные элементы смежного класса $F_{q}^{*} \backslash\left(F_{q}^{*}\right)^{2}$.

Пусть теперь $m$ - произвольное нечетное число. Как и раньше $d=(n, q-1)$. Мы уже доказали, что существуют элементы $c_{0}^{\prime}, \ldots, c_{d-1}^{\prime} \in F_{q}$ такие, что определитель $d \times d$ матрицы $c^{\prime}$ вида (14) равен -1 . Положим $c_{0}=c_{0}^{\prime}, c_{m}=c_{1}^{\prime}, \ldots$, $c_{(d-1) m}=c_{d-1}^{\prime}$ и $c_{i}=0$ при $i \neq 0 \bmod m(0 \leqslant i \leqslant n-1)$. Несложное рассуждение показывает, что для соответствуюшей $n \times n$ матрицы $c$

$$
\operatorname{det}(c)=\left(\operatorname{det}\left(c^{\prime}\right)\right)^{m}=(-1)^{m}=-1 .
$$

На этом заканчивается обоснование примера 6. Много других примеров можно найти в работе [15].

Теперь мы рассмотрим конструкции, позволяюшие по уже имеюшимся неприводимым С-группам строить другие неприводимые С-групшы.

ПРЕДЛОЖЕНИЕ 2. Предположим, то $\bar{G}$ - центральное расиирение группь $G$. Если $G$ - ориентируемая неприводимая С-группа, то $\bar{G}$ в том и только том случае является неприводимой ориентируемой $\mathrm{C}$-группой, когда естественное отображение $\bar{G} \rightarrow G$ индуцирует изоморфизм $\bar{G}_{a b} \cong G_{a b}$.

ДокАЗАтЕльСтво. Предположим, что $\bar{G}_{a b} \cong G_{a b}$, и пусть $\widehat{G}$ - универсальное центральное расширение группы $G$. Тогда сушествует морфизм расширений $f: \widehat{G} \rightarrow \bar{G}$. Из того, что $\widehat{G}_{a b} \cong \bar{G}_{a b} \cong G_{a b}$, легко следует, что $f$ - эпиморфизм. Пусть $g$ - С-образующий для $G$. Как вытекает из следствия $4, H_{2} G$ состоит из

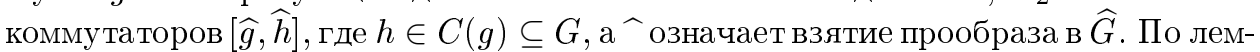
ме $6 \widehat{G}$ является С-группой. Чтобы получить $\bar{G}$ из $\widehat{G}$ надо профакторизовать $\widehat{G}$ по 
всем коммутаторам $[\widehat{g}, \widehat{h}]$, содержашимся в $\operatorname{ker} f$. Но соотношения вида $\widehat{h}^{-1} \widehat{g} \widehat{h}=\widehat{g}$ допустимы при задании С-групп. Это доказывает, что $\bar{G}-$ С-группа. В обратную сторону предложение очевидно.

Определим теперь прямое произведение в категории неприводимых ориентируемых С-групп. Сначала уточним понятие гомоморфизма. Пусть С-группы $G_{1}$ и $G_{2}$ заданы своими копредставлениями

$$
G_{i}=\left\langle X_{i} \mid R_{i}\right\rangle \quad(i=1,2) .
$$

Будем предполагать, что $X_{i}^{G_{i}}=X_{i}$. Гомоморфизм $f: G_{1} \rightarrow G_{2}$ назовем гомоморфизмом С-групп, или С-гомоморфизмом, если $f\left(X_{1}\right) \subseteq X_{2}$. Обычное прямое произведение групा $G_{1}$ и $G_{2}$ можно определить как группу $G$ вместе с эпиморфизмами $\pi_{i}: G \rightarrow G_{i}$ такими, что для любой группы $H$ и гомоморфизмов $f_{i}: H \rightarrow G_{i}$ сушествует единственный гомоморфизм $f: H \rightarrow G$, для которого диаграмма

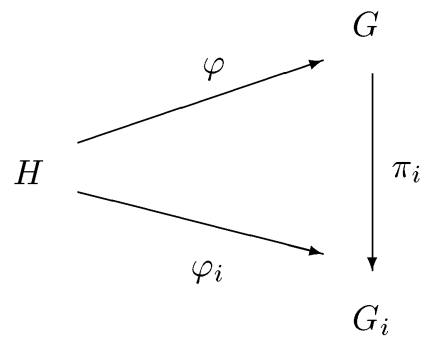

коммутативна. Прямое произведение в категории неприводимых ориентируемых С-групп определяется точно так же. Надо лишш слово "группа" заменить на "неприводимая ориентируемая С-группа" и “гомоморфизм" - на "С-гомоморфизм". Единственность такого прямого произведения следует из его универсального свойства. Докажем существование. Пусть неприводимые ориентируемые С-группы заданы копредставлениями (16). Рассмотрим группу $G$ с множеством образующих

$$
X=\left\{\left(u_{\alpha}, v_{\beta}\right) \mid u_{\alpha} \in X_{1}, v_{\beta} \in X_{2}\right\}
$$

и определяюшими соотношениями

$$
\left(u_{\alpha^{\prime}}, v_{\beta^{\prime}}\right)^{-1}\left(u_{\alpha}, v_{\beta}\right)\left(u_{\alpha^{\prime}}, v_{\beta^{\prime}}\right)=\left(u_{\alpha^{\prime}}^{-1} u_{\alpha} u_{\alpha^{\prime}}, v_{\beta}^{-1} v_{\beta} v_{\beta^{\prime}}\right) .
$$

Очевидно, $G$ - неприводимая ориентируемая С-группа. Проекции $\left(u_{\alpha}, v_{\beta}\right) \rightarrow u_{\alpha}$ и $\left(u_{\alpha}, v_{\beta}\right) \rightarrow v_{\beta}$ определяют эпиморфизмы $\pi_{i}: G \rightarrow G_{i}$. Непосредственно из определений следует, что построенная группа $G$ удовлетворяет нужному универсальному свойству. Прямое произведение в категории неприводимых С-групп будем обозначать $G_{1} \times_{\mathrm{C}} G_{2}$. Установим связь введенной конструкции с обычным прямым произведением. Для этого представим $G_{1}$ и $G_{2}$ в виде полупрямого произведения $G_{1}=\langle u\rangle K, G_{2}=\langle v\rangle L$, где $u \in X_{1}, v \in X_{2}, K=G_{1}^{\prime}, L=G_{2}^{\prime}$. Рассмотрим, далее, группу $P=\langle w\rangle N$, где $N=K \times L, w^{-1}(a, b) w=\left(u^{-1} a u, v^{-1} b v\right)$ для $a \in K, b \in L$. Множество $Y$ элементов $w\left(a_{\alpha}, b_{\beta}\right)$ таких, что $u a_{\alpha} \in X_{1}, v b_{\beta} \in X_{2}$, инвариантно, поэтому определено универсальное С-накрытие $P^{*}=(P, Y)$. 
ПРЕДЛОЖЕНИЕ 3. Отображсние $f:\left(u_{\alpha}, v_{\alpha}\right) \rightarrow w\left(u^{-1} u_{\alpha}, v^{-1} v_{\beta}\right)$ продолжсается до изоморфизма $G_{1} \times_{\mathrm{C}} G_{2} \cong P^{*}$. Ядро естественного гомоморфизма $P^{*} \rightarrow P$ изоморфно $\left(K_{a b} \otimes L_{a b}\right) \otimes\langle w\rangle \mathbb{Z}$.

ДокАЗАТЕЛьство. Непосредственно из определений следует, что отображение $f$ продолжается до гомоморфизма $f: G_{1} \times_{\mathrm{C}} G_{2} \rightarrow P$, а благодаря универсальному свойству С-накрытия $f$ продолжается до гомоморфизма $f^{*}: G_{1} \times{ }_{\mathrm{C}} G_{2} \rightarrow P^{*}$. Теперь заметим, что определены эпиморфизмы $\varphi_{i}: P \rightarrow G_{i}$, где $\varphi_{1}\left(w\left(a_{\alpha}, b_{\beta}\right)\right)=$ $u a_{\alpha}, \varphi_{2}\left(w\left(a_{\alpha}, b_{\beta}\right)\right)=v b_{\beta}$, следовательно, определены эпиморфизмы $\varphi_{i}^{*}: P^{*} \rightarrow G_{i}$. Тогда по универсальному свойству прямого произведения определен гомоморфизм $\varphi: P^{*} \rightarrow G_{1} \times_{\mathrm{C}} G_{2}$, который и дает отображение обратное к $f^{*}$. Вычислим ядро отображения $P^{*} \rightarrow P$. По теореме 4 оно совпадает с коядром отображения $\rho_{w}: C(w) \rightarrow H_{2}(P)$. Из спектральной последовательности расширения

$$
1 \longrightarrow K \times L \longrightarrow P \longrightarrow\langle w\rangle \longrightarrow 1
$$

и формулы Кюннета для прямого произведения $K \times L$ легко следует, что

$$
H_{2} P=H_{2} G_{1} \oplus H_{2} G_{2} \oplus\left(H_{1} K \otimes H_{1} L\right) \otimes_{\langle w\rangle} \mathbb{Z}
$$

Так как $G_{1}$ и $G_{2}$ - неприводимые С-группы, образ отображений $\rho_{u}$ и $\rho_{v}$ совпадает, соответственно, с $H_{2} G_{1}$ и $H_{2} G_{2}$, но $\operatorname{Im} \rho_{w}=\operatorname{Im} \rho_{u} \oplus \operatorname{Im} \rho_{v}$, следовательно, Coker $\rho_{w}=\left(K_{a b} \oplus L_{a b}\right) \otimes\langle w\rangle \mathbb{Z}$.

Пример 7. Пусть $G=\left\langle w, a \mid w^{-1} a w=a^{-1}, a^{3}=1\right\rangle$. Очевидно, $G^{\prime} \cong \mathbb{Z}_{3}-$ циклическая группа порядка 3 . Из следствия 4 вытекает, что $G$ - неприводимая ориентируемая С-группа (с множеством С-образующих $w, w a, w a^{2}$ ). Рассмотрим прямое произведение $G \times{ }_{\mathrm{C}} G$. Тогда ядро соответствуюшего отображения $P^{*} \rightarrow P$ изоморфно $\left(\mathbb{Z}_{3} \otimes \mathbb{Z}_{3}\right) \otimes\langle w\rangle \mathbb{Z}$, а так как $(a \otimes a) w=(-a) \otimes(-a)=a \otimes a$, то ядро изоморфно $\mathbb{Z}_{3}$. Итак, коммутант прямого произведения $G \times{ }_{\mathrm{C}} G$ - центральное (неабелево) расширение прямого произведения $\mathbb{Z}_{3} \times \mathbb{Z}_{3}$ с помошью циклической групшы $\mathbb{Z}_{3}$.

Наконец, введем свободное произведение. Пусть неприводимые ориентируемые С-группы $G_{1}$ и $G_{2}$ заданы своими копредставлениями (16). Их свободным произведением $G_{1} *_{\mathrm{C}} G_{2}$ в категории неприводимых ориентируемых С-групп назовем факторгруппу обычного свободного произведения $G_{1} * G_{2}$ по соотношению $u=v$, где $u \in X_{1}, v \in X_{2}$. Очевидно, $G_{1} *_{\mathrm{C}} G_{2}-$ С-группа. Имеются вложения $\mu_{i}: G_{i} \rightarrow G$ $(i=1,2), G^{\prime} \cong G_{1}^{\prime} * G_{2}^{\prime}$ и действие образующего $u=v$ на $G^{\prime}$ согласовано с действием $u$ на $G_{1}^{\prime}$ и $v$ на $G_{2}^{\prime}$. Для любой неприводимой ориентируемой С-группы $H$ и гомоморфизмов $\varphi_{i}: G_{i} \rightarrow H$ таких, что $\varphi_{1}(u)=\varphi_{2}(v)$, сушествует единственнњй 
гомоморфизм $\varphi: G \rightarrow H$ такой, что диаграмма

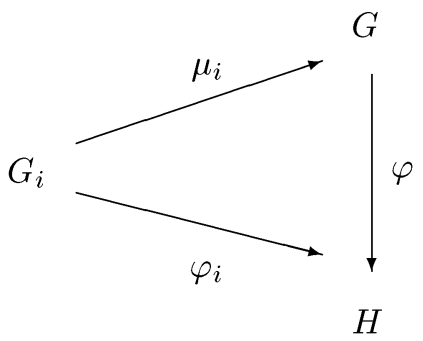

коммутативна. Если $u^{\prime} \in X_{1}, v^{\prime} \in X_{2}$ - два других образующих, то $u^{\prime}$ сопряжен с $u$, а $v^{\prime}-$ с $v$, поэтому свободное произведение $G_{1} *_{\mathrm{C}} G_{2}$ определено с точностью до автоморфизма, ограничения которого на $G_{1}$ и $G_{2}$ - внутренние автоморфизмы. Неоднозначность в выборе отождествляемых образующих похожа на произвол в выборе вырезаемых окрестностей при построении связной суммы многообразий.

\section{Список литературы}

1. Kervaire $M$. On higher dimensional knots // Differencial and combinatorial Topology: Princeton Univ. Press, 1965. P. 105-120.

2. Kulikov Vic. S., Kuzmin Yu. V. On the fundumental groups of the complements to codimension 2 submanifolds of sphere // LMS lectures series (to appear).

3. Simon J. Wirtinger approximations and the knot groups of $S^{n}$ in $S^{n+2} / /$ Pac. J. Math. 1980. V. 90. P. $177-190$.

4. Hausmann J., Kervaire M. Sous-gropes derives gropes de noeud // L'Enseignement M. 1978. V. 24. P. 111-123.

5. Suzuki S. Problems of 2-spheres in 4-sphere // Math. Sem. Notes. Kobe University. 1976. V. 4. № 3 .

6. Hillman J. 2-knots and their groups: Cambridge Univ. Press, 1989.

7. Howie J. On the asphericiy of ribbon disk complements // Trans. Amer. Math. Soc. 1985. V. 289. P. 281-302.

8. Кроуәл P., Фокс Р. Введение в теорию узлов. М.: Мир, 1985.

9. Куликов Вик. С. Геометрическая реализация С-групп // Изв. РАН. Сер. матем. 1994. T. 58. № 4. C. 194-204.

10. Gilbert N., Howie J. LOG groups, higher knot groups and Schur multipiers // Bull. London Math. Soc. (to appear).

11. Кузвмин Ю. В. Об одном способе построения С-групп // Изв. РАН. Сер. матем. 1995. T. 4. C. $105-124$.

12. Милнор Дж. Введение в алгебраическую К-теорию. М.: Мир, 1974.

13. Горенстейн Д. Конечные простые группы. М.: Мир, 1985.

14. Klyachko A. A. A funny property of sphere and equations over groups // Comm. Algebra. 1993. V. 21. № 7. P. 2555-2575.

15. Семенов Ю. С. О коммутантах неприводимых С-групп // Матем. сб. 1996. Т. 187. № 3 (в печати). 